\title{
On Alternating Quantum Walks
}

\author{
Jenia Rousseva, Yevgeniy Kovchegov
}

\begin{abstract}
We study an inhomogeneous quantum walk on a line that evolves according to alternating coins, each a rotation matrix. For the quantum walk with the coin alternating between clockwise and counterclockwise rotations by the same angle, we derive a closed form solution for the propagation of probabilities, and provide its asymptotic approximation via the method of stationary phase. Finally, we observe that for a $\frac{\pi}{4}$ angle, this alternating rotation walk will replicate the renown Hadamard walk.

Keywords: quantum walk

2010 MSC: 81P45, 81P68
\end{abstract}

\section{Introduction}

A quantum walk is the quantum mechanical analog of the classical random walk, a stochastic model following the motion of a particle along a graph. Historically, classical walks have been successful in the design efficient computer algorithms for traditional computers. With the rise of quantum computing, quantum walks have been used to create new algorithms, such as Grover's search algorithm and Shor's factorization algorithm which provide quadratic and possibly exponential speedup, respectively, over their classical counterparts. Recently, 1] proved that quantum walks are universal for quantum computation, meaning that any quantum algorithm can be reconstructed as a quantum walk algorithm. With this in mind, quantum walks merit further research about their

Email addresses: jrousseva@eagles.ewu.edu (Jenia Rousseva), kovchegy@math . oregonstate. edu (Yevgeniy Kovchegov)

Preprint submitted to Physica A: Statistical Mechanics and its ApplicationsOctober 30, 2016

(C) 2016. This manuscript version is made available under the Elsevier user license http://www.elsevier.com/open-access/userlicense/1.0/ 
unique properties.

The two main types of quantum walks are discrete-time versus continuous time quantum walks 2, 3, 4. While significant work has been done on both types of walks, for the purpose of this paper we will focus on discrete-time quantum walks. Perhaps, the most well-known of these types of quantum walks is the Hadamard walk. Nayak and Viswanath [5] outline the dynamics of the Hadamard walk and also derive the asymptotic functional form of its corresponding probability distribution.

Consequently, numerous other studies have analyzed the behavior of the wave function governing the evolution of the quantum walker by looking into the features of the coin operator. Montero [6] was able to obtain closedform formulas for the evolution of the wave function for a unidirectional quantum walk on an infinite line with an arbitrary coin operator. It has even been shown that all nonequivalent quantum walks can be reduced to a singleparameter family of quantum walks [7. In recent years, a special family of quantum walks called inhomogeneous quantum walks have received considerable attention. Inhomogeneous quantum walks may further categorized as either space-inhomogeneous, i.e. the coin operator varies with the position of the walker, or time-inhomogeneous where a different coin operator is used at different times. For space-inhomogeneous walks with a periodic coin operator, Linden and Sharam demonstrate that depending on the period, these walks may be bounded or unbounded in time [8]. There has also been interest in modeling the behavior of classical random walks through quantum walks. Typically, this behavior can be recovered in the asymptotic limit of quantum walks by destroying the coherence of the pure state in the quantum system 9 . However, Montero 9] shows that classical-like walk can be mimicked at all time scales by an inhomogeneous quantum walk with a time-dependent coin operator. Finally, in [10, a class of space and time inhomogeneous quantum walks on a line is considered. There, a remarkable $U(1)$ gauge invariance is observed, and a continuous limit of these quantum walks is investigated.

Continuing with the topic of inhomogeneous quantum walks, in this paper 
we explore a discrete-time one dimensional quantum walk which is both spaceand time- inhomogeneous. In particular, our coin operator varies at even and odd times/positions, so we call this type of walk an alternating quantum walk. For a subclass of alternating quantum walks that we call the rotations walks, in which the coin alternates between clockwise and counterclockwise rotations by the same angle $\theta$ we obtain a closed-form expression for the coefficients of the wave function and perform asymptotic analysis to arrive at a formula for the walk's probability distribution.

Before, we delve into the analysis of our quantum walk, we present a brief overview on the construction of a quantum walk.

For a one-dimensional quantum walk, we allow a particle to move on the integer points of the line and at each time step, the spin or chirality of the particle undergoes a unitary transformation and then the particle moves accordingly to the left or right or a superposition of these chirality states. The quantum walk model for discrete-time walks consists of two quantum mechanical systems: a walker and a coin, and an evolution operator that is applied to both systems at discrete times only [2]. The walker keeps track of the particle's position and is recorded as a vector in a Hilbert space of infinite, but countable dimension denoted $\mathcal{H}_{p}=\{|j\rangle ; j \in \mathbb{Z}\}$. The coin space is two-dimensional Hilbert space denoted $\mathcal{H}_{c}$, spanned by the two basis states $\{|\uparrow\rangle,|\downarrow\rangle\}$ where $|\uparrow\rangle=\left(\begin{array}{ll}1 & 0\end{array}\right)^{T}$ and $|\downarrow\rangle=\left(\begin{array}{ll}0 & 1\end{array}\right)^{T}$. The state of the total system is contained in the space $\mathcal{H}=\mathcal{H}_{c} \otimes \mathcal{H}_{p}$

We model the state of the total system using the wave function. Hence, we define $\left|\psi_{j}(t)\right\rangle=a_{j}(t)|\uparrow\rangle+b_{j}(t)|\downarrow\rangle$ to be the wave function at a given position $j$ and time $t$ with coefficients $a_{j}(t)$ and $b_{j}(t)$. Then, $|\psi(t)\rangle=\sum_{j \in \mathbb{Z}}\left|\psi_{j}(t)\right\rangle$ is the total wave function over all integers at time $t$ Finally, the total probability of the particle being at $j$ during time $t$ is $P_{j}(t)=\left|a_{j}(t)\right|^{2}+\left|b_{j}(t)\right|^{2}$.

To perform a quantum walk, we begin with our initial state $|\psi(0)\rangle$. We may continue to apply this transformation, multiplying by $\mathrm{U}$ each time, which means that $|\psi(t)\rangle=U^{t}|\psi(0)\rangle$, where $t$ represents the number of time steps. 
The transformation $U$ is defined as

$$
U=S \cdot(C \otimes I) .
$$

where $C$ represents the coin operator, $I$ is the identity matrix in the position space and $S$ is the conditional shift operator defined as

$$
S=\sum_{j \in \mathbb{Z}}|\uparrow\rangle\langle\uparrow|\otimes| j+1\rangle\left\langle j\left|+\sum_{j \in \mathbb{Z}}\right| \downarrow\right\rangle\langle\downarrow|\otimes| j-1\rangle\langle j| .
$$

$S$ shifts the position of the particle by one unit to the left or to the right. If the spin is up, then $S$ will move the particle to the right (from $j$ to $j+1$ ) and a down spin will lead $S$ to move the particle to the left (from $j$ to $j-1$ ).

The rest of the paper is organized as follows: Section 2 presents our main results from the rotations walk and the Appendix gives further technical details and numerical calculations.

\section{Results and Discussion}

In this section, we present the results for our alternating quantum walk. Since at even times, the coin operator is a clockwise rotation and at odd times we use a counter-clockwise rotation, we call this quantum walk the rotations walk.

\subsection{The Rotations Walk}

\subsubsection{Definition}

We define our rotations walk such that $C_{\text {odd }}=\left(C_{\text {even }}\right)^{-1}$, where the coin operator $C_{\text {odd }}$ will be used at odd times and $C_{\text {even }}$ is to be applied at even time steps. More specifically, we have

$$
C_{\text {even }}=\left(\begin{array}{cc}
\cos (\theta) & -\sin (\theta) \\
\sin (\theta) & \cos (\theta)
\end{array}\right) \quad \text { and } \quad C_{\text {odd }}=\left(\begin{array}{cc}
\cos (\theta) & \sin (\theta) \\
-\sin (\theta) & \cos (\theta)
\end{array}\right),
$$

where $\theta$ is a fixed constant and $\theta \in[0,2 \pi]$. 


\subsubsection{Recursion Formulas and Fourier Transform}

Using the wave function and the definition of a quantum walk, we find recursion formulas for both $a_{j}(t+1)$ and $b_{j}(t+1)$ from $a_{j}(t)$ and $b_{j}(t)$, respectively, for both coin operators.

For $C_{\text {even }}$, this gives for $j \in \mathbb{Z}$,

$$
\begin{aligned}
& a_{j}(t+1)=\cos (\theta) a_{j-1}(t)-\sin (\theta) b_{j-1}(t) \\
& b_{j}(t+1)=\sin (\theta) a_{j+1}(t)+\cos (\theta) b_{j+1}(t) .
\end{aligned}
$$

Similarly, for $C_{\text {odd }}$ we get for $j \in \mathbb{Z}$,

$$
\begin{aligned}
& a_{j}(t+1)=\cos (\theta) a_{j-1}(t)+\sin (\theta) b_{j-1}(t) \\
& b_{j}(t+1)=-\sin (\theta) a_{j+1}(t)+\cos (\theta) b_{j+1}(t) .
\end{aligned}
$$

We take the Fourier transform of the recursion formulas in order to express the recursion with a $2 \times 2$ matrix. After performing a Fourier transform the recurrence relations for the walk at even times, we may express the transformed $\hat{a}_{t+1}(s)$ and $\hat{b}_{t+1}(s)$ in the following manner:

$$
\left(\begin{array}{l}
\hat{a}_{t+1}(s) \\
\hat{b}_{t+1}(s)
\end{array}\right)=M_{e}\left(\begin{array}{l}
\hat{a}_{t}(s) \\
\hat{b}_{t}(s)
\end{array}\right), \quad \text { where } \quad M_{e}=\left(\begin{array}{cc}
\cos (\theta) e^{-i s} & -\sin (\theta) e^{-i s} \\
\sin (\theta) e^{i s} & \cos (\theta) e^{i s}
\end{array}\right)
$$

and

$$
\left(\begin{array}{c}
\hat{a}_{t+1}(s) \\
\hat{b}_{t+1}(s)
\end{array}\right)=M_{o}\left(\begin{array}{c}
\hat{a}_{t}(s) \\
\hat{b}_{t}(s)
\end{array}\right) \text {, where } \quad M_{o}=\left(\begin{array}{cc}
\cos (\theta) e^{-i s} & \sin (\theta) e^{-i s} \\
-\sin (\theta) e^{i s} & \cos (\theta) e^{i s}
\end{array}\right) .
$$

Although we apply the operators in alternating order ${ }^{1}$ we may still write them as:

$$
M^{t}=\left(M_{o} M_{e}\right)^{t / 2} \quad(\mathrm{t} \text { even }) \quad \text { and } \quad M^{t}=M_{e}\left(M_{o} M_{e}\right)^{(t-1) / 2} \quad(\mathrm{t} \text { odd })
$$

\footnotetext{
${ }^{1}$ Note that the operators $M_{e}$ and $M_{o}$ are non-commutative.
} 
where

$$
M_{o} M_{e}=\left(\begin{array}{cc}
e^{-2 i s} \cos ^{2}(\theta)+\sin ^{2}(\theta) & -\cos (\theta) \sin (\theta)\left(e^{-2 i s}-1\right) \\
\cos (\theta) \sin (\theta)\left(e^{2 i s}-1\right) & e^{2 i s} \cos ^{2}(\theta)+\sin ^{2}(\theta)
\end{array}\right) .
$$

Given initial states $\hat{a}_{0}(s)$ and $\hat{b}_{0}(s)$ and the matrix $M_{o} M_{e}$ as defined above, we define

$$
\left(\begin{array}{l}
\hat{a}_{t}(s) \\
\hat{b}_{t}(s)
\end{array}\right)=\left(M_{o} M_{e}\right)^{t / 2}\left(\begin{array}{l}
\hat{a}_{0}(s) \\
\hat{b}_{0}(s)
\end{array}\right)
$$

for even $t$. For convenience let us rename $M:=M_{o} M_{e}$.

\subsubsection{Formulas for $\hat{a}_{t}(s)$ and $\hat{b}_{t}(s)$}

Subsequently, we diagonalize the matrix (5) obtaining its eigenvalues $\lambda_{1,2}=$

$e^{ \pm i \omega_{s}}$, where $\omega_{s}$ is the angle in $\left[\frac{-\pi}{2}, \frac{\pi}{2}\right]$ with $\omega_{s}=2 \arcsin (\sin (s) \cos (\theta))$ uniquely defined. The corresponding eigenvectors and further details on how these eigenvalues were derived are presented in B.2. Now we choose an initial condition of $|\uparrow\rangle \otimes|0\rangle$; that is, the particle begins its walk at the origin oriented to the right. Hence, after performing the matrix multiplication and simplifying, we arrive at:

$$
\hat{a}_{t}(s)=\frac{1}{2}\left[\left(1-\frac{\cos (s) \cos (\theta)}{\sqrt{1-A}}\right) e^{i \omega_{s} t / 2}+\left(1+\frac{\cos (s) \cos (\theta)}{\sqrt{1-A}}\right) e^{-i \omega_{s} t / 2}\right]
$$

and

$$
\hat{b}_{t}(s)=\frac{e^{i s} \sin (\theta)}{2 \sqrt{1-A}}\left(e^{i \omega_{s} t / 2}-e^{-i \omega_{s} t / 2}\right)
$$

for all even $t$, where we let $A=\sin ^{2}(s) \cos ^{2}(\theta)$. The expressions for $\hat{a}_{t}(s)$ and $\hat{b}_{t}(s)$ when $t$ is odd are obtained via 44 from the above formulas (7) and (8).

\subsubsection{Closed-form Formulas for $a_{j}(t)$ and $b_{j}(t)$}

Finally, we take the inverse Fourier transform of (7) and (8) to arrive at final closed-form formulas for $a_{j}(t)$ and $b_{j}(t)$ when $t$ is even:

$$
a_{j}(t)=\frac{1+(-1)^{j+t}}{2} \int_{-\pi}^{\pi} \frac{d s}{2 \pi}\left(1-\frac{\cos (s) \cos (\theta)}{\sqrt{1-A}}\right) e^{i\left(j s+\omega_{s} t / 2\right)}
$$




$$
b_{j}(t)=\frac{1+(-1)^{j+t}}{2} \int_{-\pi}^{\pi} \frac{d s}{2 \pi} \frac{e^{i s} \sin (\theta)}{\sqrt{1-A}} e^{i\left(j s+\omega_{s} t / 2\right)}
$$

Next, we combine the equations (9) and (10) with $(2)$ and (3) to obtain the following closed-form formulas for $a_{j}(t)$ and $b_{j}(t)$ when $t$ is odd: the expression for $b_{j}(t)$ will be the same as in 10 , while

$$
a_{j}(t)=\frac{1+(-1)^{j+t}}{2} \int_{-\pi}^{\pi} \frac{d s}{2 \pi}\left(-1+\frac{\cos (s) \cos (\theta)}{\sqrt{1-A}}\right) e^{i\left(j s+\omega_{s} t / 2\right)}
$$

Thus, we observe the following peculiar behavior of the rotations walk. If the rotations walk begins at $|\uparrow\rangle \otimes|0\rangle$, the expression for $b_{j}(t)$ will be the same

for all $t$ whether odd or even, while the expression for $a_{j}(t)$ will differ for odd and even values of $t$ by alternating the sign. Here, the general formula for $a_{j}(t)$ can be written as follows

$$
a_{j}(t)=\frac{(-1)^{t}+(-1)^{j}}{2} \int_{-\pi}^{\pi} \frac{d s}{2 \pi}\left(1-\frac{\cos (s) \cos (\theta)}{\sqrt{1-A}}\right) e^{i\left(j s+\omega_{s} t / 2\right)} \quad(t=0,1, \ldots) .
$$

\subsubsection{Probability Distribution}

As the aforementioned integrals cannot be exactly calculated, we approximate the integrals asymptotically using the method of stationary phase (refer to Appendix A.2. Applying this method, we find the following asymptotic expressions:

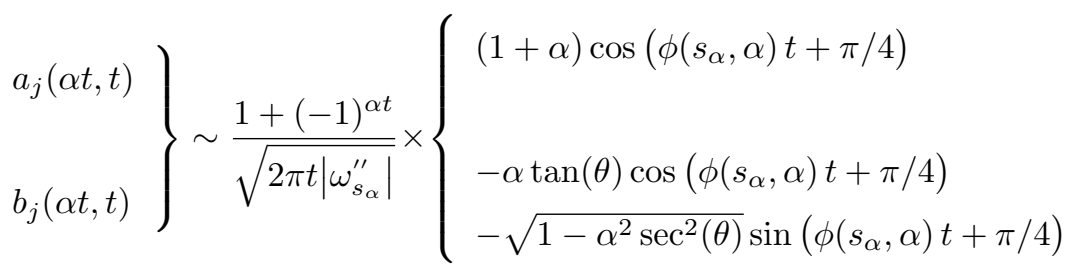

where we substituted $j=\alpha t$ for $\alpha \in[-1,1]$.

Therefore, we can calculate the probability of observing the particle at any point $j=\alpha t$. The asymptotic distribution for points $\alpha=j / t$ between 
$-\cos (\theta)+\epsilon$ and $\cos (\theta)-\epsilon$, for any small constant $\epsilon>0$ is

$$
P(\alpha, t)=|a(\alpha t, t)|^{2}+|b(\alpha t, t)|^{2} .
$$

\subsection{Alternative Representation of the Hadamard Walk}

Our numerical calculations (see Figure 1) suggest that for $\theta=\frac{\pi}{4}$, the alternating rotations walk collapses exactly into famous the Hadamard walk. This was a rather unintuitive, so we aim to prove it analytically.

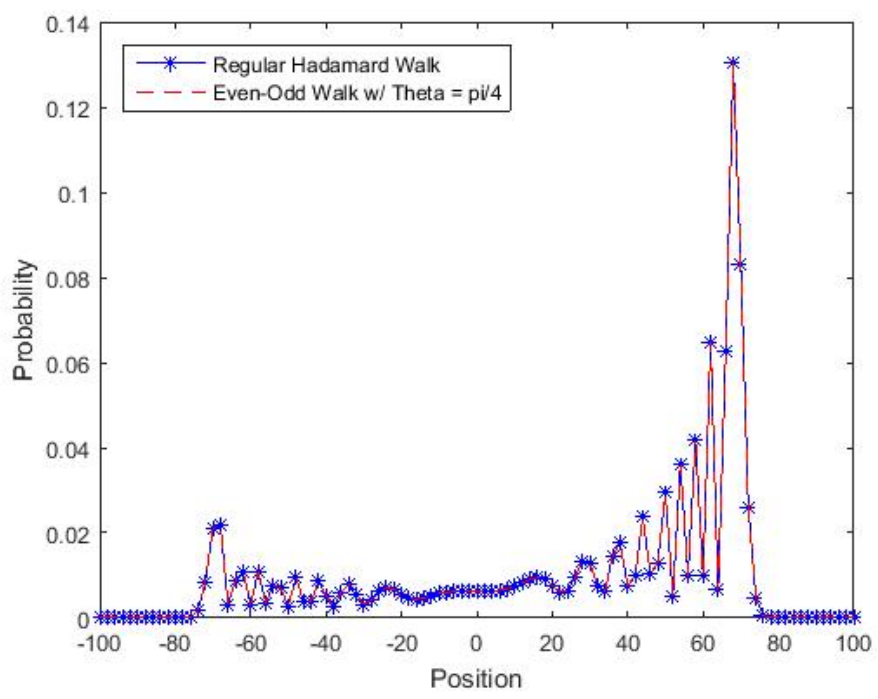

Figure 1: The probability distributions of the single-coined Hadamard walk and our doublecoined alternating rotations walk match exactly.

Nayak and Vishwanath 5 found closed-form integral formulas for the coefficients $a_{j}(t)$ and $b_{j}(t)$ which are presented in $(15)$ and (16). There, for the Hadamard walk with the initial condition $|\uparrow\rangle \otimes|0\rangle$,

$$
a_{j}(t)=\frac{1+(-1)^{j+t}}{4 \pi} \int_{-\pi}^{\pi} \frac{e^{-i s}}{\sqrt{1+\cos ^{2}(s)}} e^{i\left(j s-\omega_{s} t\right)} d s
$$

and

$$
b_{j}(t)=\frac{1+(-1)^{j+t}}{4 \pi} \int_{-\pi}^{\pi}\left(1-\frac{\cos (s)}{\sqrt{1+\cos ^{2}(s)}}\right) e^{i\left(j s-\omega_{s} t\right)} d s .
$$


To do this, we first substitute $-j$ for $j$ into both 15 and $(16)$ and then take the conjugate of the entire integral expression. We can do this because the amplitudes are real since the entries in the coin operators are real which implies that $\overline{a_{j}(t)}=a_{j}(t)$ and $\overline{b_{j}(t)}=b_{j}(t)$. After these modifications, the coefficients of the wave function with initial condition of $|\uparrow\rangle \otimes|0\rangle$ for the Hadamard walk become:

$$
\begin{gathered}
a_{j}(t)=\frac{1+(-1)^{j+t}}{2} \int_{-\pi}^{\pi} \frac{d s}{2 \pi}\left(1-\frac{\cos (s)}{\sqrt{1+\cos ^{2}(s)}}\right) e^{i\left(j s+\widetilde{\omega_{s}} t\right)} \\
b_{j}(t)=\frac{1+(-1)^{j+t}}{2} \int_{-\pi}^{\pi} \frac{d s}{2 \pi} \frac{e^{i s}}{\sqrt{1+\cos ^{2}(s)}} e^{i\left(j s+\widetilde{\omega_{s}} t\right)}
\end{gathered}
$$

where $\sin \left(\widetilde{\omega_{s}}\right)=\frac{-\sin (s)}{\sqrt{2}}$. We use $\widetilde{\omega_{s}}$ in place of $\omega_{s}$ to distinguish from the $\omega_{s}$ in 12 and 10 .

We have defined $\omega_{s}=2 \arcsin (\sin (s) \cos (\theta))$, so we have $\cos \left(\omega_{s}\right)=1-$ $2 \sin ^{2}(s) \cos ^{2}(\theta)$. If we substitute $\theta=\pi / 4$ into the expression for $\omega_{s}$, we get $\cos \left(\omega_{s}\right)=1-2 \sin ^{2}(s) \cos ^{2}\left(\frac{\pi}{4}\right)=1-2\left(\frac{1}{\sqrt{2}}\right)^{2} \sin ^{2}(s)=1-\sin ^{2}(s)=\cos ^{2}(s)$. Thus, $\cos \left(\omega_{s}\right)=\cos ^{2}(s)$.

Next, we observe that $\cos \left(2 \widetilde{\omega_{s}}\right)=1-2 \sin ^{2}\left(\widetilde{\omega_{s}}\right)=1-\frac{2 \sin ^{2}(s)}{2}=1-\sin ^{2}(s)=$ $\cos ^{2}(s)$. Hence, we have that $\cos \left(\omega_{s}\right)=\cos \left(\widetilde{\omega_{s}}\right) \Rightarrow \widetilde{\omega_{s}}=\frac{\omega_{s}}{2}$.

Substituting $\theta=\pi / 4$ into 12 , we obtain

$$
\begin{aligned}
a_{j}(t) & =\frac{(-1)^{t}+(-1)^{j}}{2} \int_{-\pi}^{\pi} \frac{d s}{2 \pi}\left(1-\frac{\frac{1}{\sqrt{2}} \cos (s)}{\sqrt{1-\frac{1}{2}\left(1-\cos ^{2}(s)\right)}}\right) e^{i\left(j s+\omega_{s} t / 2\right)} \\
& =\frac{(-1)^{t}+(-1)^{j}}{2} \int_{-\pi}^{\pi} \frac{d s}{2 \pi}\left(1-\frac{\cos (s)}{\sqrt{1+\cos ^{2}(s)}}\right) e^{i\left(j s+\left(\frac{\omega_{s}}{2}\right) t\right)}
\end{aligned}
$$

which, when we replace $\frac{\omega_{s}}{2}$ with $\widetilde{\omega_{s}}$, will match (17) up to a sign in front of the expression. 
Substituting $\theta=\pi / 4$ into 10$]$, we obtain

$$
\begin{aligned}
b_{j}(t) & =\frac{1+(-1)^{j+t}}{2} \int_{-\pi}^{\pi} \frac{d s}{2 \pi} \frac{\frac{1}{\sqrt{2}} e^{i s}}{\sqrt{\frac{1}{2}\left(1+\cos ^{2}(s)\right)}} e^{i\left(j s+\omega_{s} t / 2\right)} \\
& =\frac{1+(-1)^{j+t}}{2} \int_{-\pi}^{\pi} \frac{d s}{2 \pi} \frac{e^{i s}}{\sqrt{1+\cos ^{2}(s)}} e^{i\left(j s+\left(\frac{\omega_{s}}{2}\right) t\right)} .
\end{aligned}
$$

This is exactly $(18)$ after replacing $\frac{\omega_{s}}{2}$ with $\widetilde{\omega_{s}}$.

Therefore, we have shown that the Hadamard walk, a walk with one coin operator, can be replicated with two coins when $\theta=\frac{\pi}{4}$ in the rotations walk.

\subsection{Discussion}

Many aspects of our research could be further generalized. We worked only with the same initial state, $|\uparrow\rangle \otimes|0\rangle$. Similarly, there are many ways to make a coin space-inhomogeneous. For example, we could define a coin that is different at every point on the line. We could also study a walk that is strictly time-inhomogeneous, independent of position, and compare results with similar space-inhomogeneous walks. Learning to predict and control the behavior of quantum walks using these variables could make them even more powerful tools in computing and other applications.

The discovery that the rotations walk could replicate the Hadamard walk raises several poignant questions. Can all homogeneous walks be modeled by inhomogeneous walks? Methods to control walks by adjusting their parameters could lead to insights for answering these questions.

\section{Acknowledgments}

The authors would like to thank Autumn Asay for her participation in this project. We would also like to acknowledge that this work was done as part of the Summer 2015 REU Program in Mathematics and Theoretical Computer Sci-

ence at Oregon State University under the sponsorship of the National Science Foundation (Grant DMS-1359173). 


\section{References}

[1] A. M. Childs, Universal computation by quantum walk, Phys. Rev. Lett. 102. arXiv:0806.1972v1, doi:10.1103/PhysRevLett.102.180501

[2] S. E. Venegas-Andraca, Quantum walks: a comprehensive review, Quantum Information Processing 11 (2012) 1015-1106. arXiv:1201.4780v2, doi:10.1007/s11128-012-0432-5.

[3] Z. Dimcovic, D. Rockwell, I. Milligan, R. Burton, T. Nguyen, Y. Kovchegov, Framework for discrete-time quantum walks and a symmetric walk on a binary tree, Physical Review A 84 (3). doi:10.1103/PhysRevA.84.032311.

[4] Z. Dimcovic, Discrete-time quantum walks via interchange framework and memory in quantum evolution, Ph.D. thesis, Oregon State University, Kidder Hall 368, Oregon State University, Corvallis, OR 97331-4605 (6 2012).

[5] A. Nayak, A. Vishwanath, Quantum walk on the line, arXiv preprint quant$\mathrm{ph} / 0010117$.

[6] M. Montero, Quantum walk with a general coin: Exact solution and asymptotic properties, Quantum Information Processing 14 (2014) 839866. arXiv:1310.7486v3, doi:10.1007/s11128-014-0908-6.

[7] S. K. Goyal, T. Konrad, L. Diósi, Unitary equivalence of quantum walks, Physics Letters A 379 (2014) 100-104. arXiv:1401.0196v2, doi:10.1016/ j.physleta.2014.11.001.

[8] N. Linden, J. Sharam, Inhomogeneous quantum walks, Physical Review A 80 (5) (2009) 052327.

[9] M. Montero, Classical-like behavior in quantum walks with inhomogeneous, time-dependent coin operators, arXiv preprint arXiv:1505.07996.

[10] G. Di Molfetta, M. Brachet, F. Debbasch, Quantum walks in artificial electric and gravitational fields, Physica A: Statistical Mechanics and its Applications 397 (2014) 157-168. 


\section{A. Technical Background}

\section{A.1. The Fourier Transform}

Let $\mathrm{f}$ be a function $f: \mathbb{Z} \rightarrow \mathbb{C}$. Then, we define a Fourier transform $\hat{f}$ : $[-\pi, \pi] \rightarrow \mathbb{C}$ as:

$$
\hat{f}_{t}(s)=\sum_{j} f_{j}(t) e^{-i j s},
$$

where $i$ is the imaginary unit and $s$ is a real number, summing over all integer positions $j$. Consequently, the inverse Fourier transform is given by

$$
f_{j}(t)=\frac{1}{2 \pi} \int_{-\pi}^{\pi} \hat{f}_{t}(s) e^{i j s} d s
$$

\section{A.2. The Method of Stationary Phase}

The integral expressions for our coefficients $a_{j}(t)$ and $b_{j}(t)$ clearly cannot be solved by ordinary integration techniques, so we must consider a well-known method which allows for the asymptotic expansion of integrals called the method of stationary phase [5].

We consider an integral of the form:

$$
I(t)=\int_{a}^{b} g(s) e^{i t \phi(s)} d s
$$

as $t$ tends to infinity. We assume that the exponential term in the integral oscillates rapidly when $t$ is large and if $\phi$, called the phase of the integral, is not constant in any sub-interval. Also, if $g(s)$ is a smooth function of $s$, then terms from adjacent sub-intervals will almost cancel each other out, meaning that the major contribution to the value of the integral comes from regions where the oscillations are slow. These regions of slow oscillations occur exactly at the stationary points, i.e the points where the phase term is stationary. More precisely, the points $c$ where $\phi^{\prime}(c)=0$. Thus, the significant terms in the expansion come from a small interval around the stationary points. 
Without loss of generality, we assume that $\phi$ has exactly one stationary point occurring at the left endpoint of the interval, a. Furthermore, we make the assumption that $g$ is smooth and non-vanishing at $a$. The order of a stationary point, $c$, corresponds to the last derivative of $\phi$ in the Taylor expansion of $\phi(s)$ at $c$ which is nonzero at $c$. Suppose that the order of $a$ is $p-1$, then $\phi^{\prime}(a)=\phi^{(2)}(a)=\cdots=\phi^{(p-1)}(a)=0$, but $\phi^{(p)}(a) \neq 0$. Then, the dominant behavior of $I$ is given by

$$
I(t) \sim g(s) e^{i t \phi(s) \pm i \pi / 2 p}\left[\frac{p !}{t\left|\phi^{(p)}(a)\right|}\right]^{1 / p} \frac{\Gamma(1 / p)}{p}, \quad t \rightarrow+\infty,
$$

where we use $e^{i \pi / 2 p}\left(e^{-i \pi / 2 p}\right)$ if $\phi^{(p)}(a)>0\left(\phi^{(p)}(a)<0\right)$.

\section{B. Calculations for the Rotations Walk}

\section{B.1. Fourier Transform}

We would like to find the Fourier transform of the recursion formulas for the $C_{\text {even }}$ operator by substituting the formulas for $a_{j}(t+1)$ and $b_{j}(t+1)$ into (A.1).

$$
\begin{aligned}
\hat{a}_{t+1}(s) & =\sum_{j} a_{j}(t) e^{-i j s} \\
& =\sum_{j} \cos (\theta) a_{j-1}(t) e^{-i j s}+\sum_{j} \sin (\theta) b_{j-1}(t) e^{-i j s} \\
& =\sum_{k} \cos (\theta) a_{k}(t) e^{-i(k+1) s}+\sum_{k} \sin (\theta) b_{k}(t) e^{-i(k+1) s} \\
& =\cos (\theta) e^{-i s} \sum_{k} a_{k}(t) e^{-i k s}+\sin (\theta) e^{-i s} \sum_{k} b_{k}(t) e^{-i k s} \\
& =\cos (\theta) e^{-i s} \hat{a_{t}}(s)+\sin (\theta) e^{-i s} \hat{b_{t}}(s)
\end{aligned}
$$

By a symmetrical argument, we can find the Fourier transform of $\hat{b}_{t+1}(s)$.

$$
\hat{b}_{t+1}(s)=-\sin (\theta) e^{i s} \hat{a}_{t}(s)+\cos (\theta) e^{i s} \hat{b_{t}}(s)
$$




\section{B.2. Diagonalization}

Diagonalizing $M$ as defined in (5) involves finding its eigenvalues and a pair of orthonormal eigenvectors.

For the eigenvalues we find

$$
\lambda_{1,2}=1-2 \sin ^{2}(s) \cos ^{2}(\theta) \pm 2 i \sin (s) \cos (\theta) \sqrt{1-\sin ^{2}(s) \cos ^{2}(\theta)} .
$$

Both eigenvalues lie on the unit circle, and are conjugates of each other, so we can take the argument of each eigenvalue. Let $\lambda_{1}=e^{i \omega_{s}}$ and $\lambda_{2}=e^{-i \omega_{s}}$ where $\omega_{s}$ is the angle in $\left[\frac{-\pi}{2}, \frac{\pi}{2}\right]$ where $\omega_{s}=2 \arcsin (\sin (s) \cos (\theta))$ is uniquely defined. Additionally, we have $\cos \left(\omega_{s}\right)=1-2 \sin ^{2}(s) \cos ^{2}(\theta)$. Let $A:=\sin ^{2}(s) \cos ^{2}(\theta)$, so we have $\cos \left(\omega_{s}\right)=1-2 A$. Note the definition of $A$ from now on.

Now, we would like to find the corresponding orthonormal eigenvectors, $\overrightarrow{u_{1}}$ and $\overrightarrow{u_{2}}$, for each eigenvalue. This is accomplished by solving

$$
(M-\lambda I) \vec{v}=0
$$

for $\lambda=\lambda_{1,2}$ where $v=\left(\begin{array}{ll}\eta_{1} & \eta_{2}\end{array}\right)^{T}$.

In the process of solving (B.4) we derive the equation

$$
\left(\cos ^{2}(\theta) e^{-2 i s}+\sin ^{2}(\theta)-e^{ \pm i \omega_{s}}\right) \eta_{1}+\cos (\theta) \sin (\theta)\left(e^{-2 i s}-1\right) \eta_{2}=0 .
$$

for the eigenvalues $\lambda_{1,2}=e^{ \pm i \omega_{s}}$, respectively.

If we let $\eta_{1}=\cos (\theta) \sin (\theta)\left(e^{-2 i s}-1\right)$, solving (B.5) for $\eta_{2}$ gives $\eta_{2}=e^{ \pm i \omega_{s}}-\cos ^{2}(\theta) e^{-2 i s}-\sin ^{2}(\theta)$. After normalization, we have the following respective eigenvectors for $\lambda_{1,2}$.

$$
\overrightarrow{u_{1,2}}:=\frac{1}{\sqrt{N_{1,2}(s)}}\left(\begin{array}{c}
\cos (\theta) \sin (\theta)\left(e^{-2 i s}-1\right) \\
e^{ \pm i \omega_{s}}-\cos ^{2}(\theta) e^{-2 i s}-\sin ^{2}(\theta)
\end{array}\right)
$$

where $N_{1,2}(s):=8 A(1-A \pm \cos (s) \cos (\theta) \sqrt{1-A})$. 


\section{B.3. Finding Formulas for $\hat{a}_{t}(s)$ and $\hat{b}_{t}(s)$}

Since we assumed that the initial condition is $|\uparrow\rangle \otimes|0\rangle$, which is purely up, we know that $a_{0}(t)=\delta_{0, j}$, where $\delta$ is the Kronecker delta, so that

$$
\hat{a}_{0}(s)=\sum_{j} \delta_{0, j} e^{-i j s}=\delta_{0,0} e^{0}=1(1)=1 .
$$

Likewise, $b_{0}(t)=0$ which implies that $\hat{b}_{0}(s)=0$.

Therefore, we have that

$$
\left(\begin{array}{l}
\hat{a}_{0}(s) \\
\hat{b}_{0}(s)
\end{array}\right)=\left(\begin{array}{l}
1 \\
0
\end{array}\right)
$$

Hence, if we let $U=\left(\overrightarrow{u_{1}}, \overrightarrow{u_{2}}\right)$ and $D=\left(\begin{array}{cc}\lambda_{1} & 0 \\ 0 & \lambda_{2}\end{array}\right)$ we have the diagonalization of $M$. Then, if we substitute B.7 into the general formula in (??) and perform the matrix multiplications, then we obtain the expressions presented below.

$$
\begin{array}{r}
\hat{a}_{t}(s)=\frac{\left(\cos ^{2}(\theta) \sin ^{2}(\theta)\left(e^{-2 i s}-1\right)\left(e^{2 i s}-1\right)\right)}{N_{1}(s)} e^{i \omega_{s} t / 2} \\
+\frac{\left(\cos ^{2}(\theta) \sin ^{2}(\theta)\left(e^{-2 i s}-1\right)\left(e^{2 i s}-1\right)\right)}{N_{2}(s)} e^{-i \omega_{s} t / 2} \\
\hat{b}_{t}(s)=\frac{\cos (\theta) \sin (\theta)\left(e^{2 i s}-1\right)\left(e^{i \omega_{s}}-\cos ^{2}(\theta) e^{-2 i s}-\sin ^{2}(\theta)\right)}{N_{1}(s)} e^{i \omega_{s} t / 2} \\
+\frac{\cos (\theta) \sin (\theta)\left(e^{2 i s}-1\right)\left(e^{-i \omega_{s}}-\cos ^{2}(\theta) e^{-2 i s}-\sin ^{2}(\theta)\right)}{N_{2}(s)} e^{-i \omega_{s} t / 2}
\end{array}
$$

The formulas in $B .8$ and $B .9$ can be simplified further simplified to a form that is easier to work with. Since these are longer calculations, we only present the final forms of $\hat{a}_{t}(s)$ and $\hat{b}_{t}(s)$ here.

$$
\hat{a}_{t}(s)=\frac{1}{2}\left[\left(1-\frac{\cos (s) \cos (\theta)}{\sqrt{1-A}}\right) e^{i \omega_{s} t / 2}+\left(1+\frac{\cos (s) \cos (\theta)}{\sqrt{1-A}}\right) e^{-i \omega_{s} t / 2}\right]
$$




$$
\hat{b}_{t}(s)=\frac{e^{i s} \sin (\theta)}{2 \sqrt{1-A}}\left(e^{i \omega_{s} t / 2}-e^{-i \omega_{s} t / 2}\right)
$$

\section{B.4. Inverse Fourier Transform}

Our main objective is to find formulas for $a_{j}(t)$ and $b_{j}(t)$. This is accomplished by taking inverse Fourier transforms of (7) and (8). We substitute both (7) and (8) into (A.2) to obtain the following coefficients for the quantum walk at even positions.

$$
\begin{aligned}
& a_{j}(t)= \frac{1}{2 \pi} \int_{-\pi}^{\pi} \hat{a}_{t}(s) e^{i j s} d s \\
&=\frac{1}{2 \pi} \int_{-\pi}^{\pi} \frac{1}{2}\left[\left(1-\frac{\cos (s) \cos (\theta)}{\sqrt{1-A}}\right) e^{i \omega_{s} t / 2}+\left(1+\frac{\cos (s) \cos (\theta)}{\sqrt{1-A}}\right) e^{-i \omega_{s} t / 2}\right] e^{i j s} d s \\
&=\frac{1}{2 \pi} \int_{-\pi}^{\pi} \frac{1}{2}\left(1-\frac{\cos (s) \cos (\theta)}{\sqrt{1-A}}\right) e^{i \omega_{s} t / 2} \cdot e^{i j s} d s \\
& \quad+\frac{1}{2 \pi} \int_{-2 \pi}^{0} \frac{1}{2}\left(1+\frac{\cos (s) \cos (\theta)}{\sqrt{1-A}}\right) e^{-i \omega_{s} t / 2} \cdot e^{i j s} d s
\end{aligned}
$$

Now we perform a change of variable. Let $s^{*}:=s+\pi$. Then, we have that $s=s^{*}-\pi, d s^{*}=d s, s^{*}(-2 \pi)=-\pi$, and $s^{*}(0)=\pi$. Furthermore, it can be shown that $\omega_{s^{*}-\pi}=-\omega_{s^{*}}$ and that $\cos \left(s^{*}-\pi\right)=-\cos \left(s^{*}\right)$ and $\sin ^{2}\left(s^{*}-\pi\right)=\sin ^{2}\left(s^{*}\right)$. Using this information the second integral above may be rewritten as:

$$
\begin{aligned}
& \frac{1}{2 \pi} \int_{-\pi}^{\pi} \frac{1}{2}\left(1+\frac{\cos \left(s^{*}-\pi\right) \cos (\theta)}{\sqrt{1-\sin ^{2}\left(s^{*}-\pi\right) \cos ^{2}(\theta)}}\right) e^{-i \omega_{s^{*}-\pi} t / 2} \cdot e^{i j\left(s^{*}-\pi\right)} d s^{*} \\
& =\frac{(-1)^{j}}{2 \pi} \int_{-\pi}^{\pi} \frac{1}{2}\left(1-\frac{\cos \left(s^{*}\right) \cos (\theta)}{\sqrt{1-\sin ^{2}\left(s^{*}\right) \cos ^{2}(\theta)}}\right) e^{i\left(j s^{*}+\omega_{s^{*}} t / 2\right)} d s^{*}
\end{aligned}
$$

At this stage, rename $s:=s^{*}$ in the derivation above. Combine and simplify the two halves of the integral to get the final form of $a_{j}(t)$. 


$$
a_{j}(t)=\frac{1+(-1)^{j}}{2} \int_{-\pi}^{\pi} \frac{d s}{2 \pi}\left(1-\frac{\cos (s) \cos (\theta)}{\sqrt{1-A}}\right) e^{i\left(j s+\omega_{s} t / 2\right)}
$$

Continuing with our calculations, we derive the formula for $b_{j}(t)$ in a similar manner.

$$
\begin{aligned}
b_{j}(t) & =\frac{1}{2 \pi} \int_{-\pi}^{\pi} \hat{b}_{t}(s) e^{i j s} d s \\
& =\frac{1}{2 \pi} \int_{-\pi}^{\pi} \frac{e^{i s} \sin (\theta)}{2 \sqrt{1-A}}\left(e^{i \omega_{s} t / 2}-e^{-i \omega_{s} t / 2}\right) e^{i j s} d s \\
& =\frac{1}{2 \pi} \int_{-\pi}^{\pi} \frac{e^{i s} \sin (\theta)}{2 \sqrt{1-A}}\left(e^{i \omega_{s} t / 2} \cdot e^{i j s}\right) d s-\frac{1}{2 \pi} \int_{-2 \pi}^{0} \frac{e^{i s} \sin (\theta)}{2 \sqrt{1-A}}\left(e^{-i \omega_{s} t / 2} \cdot e^{i j s}\right) d s
\end{aligned}
$$

As before letting $s^{*}:=s+\pi$ within the second integral in the above expression allows us to reduce the integral in the following way

$$
\frac{(-1)^{j}}{2 \pi} \int_{-\pi}^{\pi} \frac{e^{i s^{*}} \sin (\theta)}{2 \sqrt{1-\sin ^{2}\left(s^{*}\right) \cos ^{2}(\theta)}} e^{i\left(j s^{*}+\omega_{s^{*}} t / 2\right)} d s^{*}
$$

Once again rename $s:=s^{*}$ in the derivation above and combine the two halves of the integral to get the final formula for $b_{j}(t)$.

$$
b_{j}(t)=\frac{1+(-1)^{j}}{2} \int_{-\pi}^{\pi} \frac{d s}{2 \pi} \frac{e^{i s} \sin (\theta)}{\sqrt{1-A}} e^{i\left(j s+\omega_{s} t / 2\right)}
$$

We observe that for odd positions of $j$, the amplitudes of both $a_{j}(t)$ and $b_{j}(t)$ cancel out, as desired.

Thus, we have obtained two integrals that describe the coefficients of the wave function at a position $j$ and time $t$. Therefore, we calculate the probability of being at $j$ at time $t$ as $P_{j}(t)=\left|a_{j}(t)\right|^{2}+\left|b_{j}(t)\right|^{2}$.

\section{B.5. Asymptotic Expansion of $a_{j}(t)$ and $b_{j}(t)$}

Using the method of stationary phase, described in A.2, we would like to asymptotically expand the integrals 12 and 10 in order to analyze the 
behavior of the wave function as $t$ tends to $+\infty$. To do this, we consider an integral of the form:

$$
I(\alpha, t)=\int_{-\pi}^{\pi} \frac{d s}{2 \pi} g(s) e^{i \phi(s, \alpha) t}
$$

If we substitute $j=\alpha t$ for $\alpha \in[-1,1]$ into the expressions in 12$]$ and 10$]$, we can obtain integrals in the form of B.10. After doing so we can defint ${ }^{2}$

$$
\begin{array}{ll}
I_{1}(\alpha, t):=\frac{1+(-1)^{\alpha t}}{2} \int_{-\pi}^{\pi} \frac{d s}{2 \pi}\left(1-\frac{\cos (s) \cos (\theta)}{\sqrt{1-A}}\right) e^{i t\left(\alpha s+\omega_{s} / 2\right)} & \text { for } a_{j}(t) \\
I_{2}(\alpha, t):=\frac{1+(-1)^{\alpha t}}{2} \int_{-\pi}^{\pi} \frac{d s}{2 \pi} \frac{e^{i s} \sin (\theta)}{\sqrt{1-A}} e^{i t\left(\alpha s+\omega_{s} / 2\right)} & \text { for } b_{j}(t)
\end{array}
$$

Furthermore, we can let

$$
\begin{aligned}
& g_{1}(s):=\left(1-\frac{\cos (s) \cos (\theta)}{\sqrt{1-A}}\right), \\
& g_{2}(s):=\frac{e^{i s} \sin (\theta)}{\sqrt{1-A}}
\end{aligned}
$$

and since both $I_{1}$ and $I_{2}$ have the same phase term, define

$$
\phi(s, \alpha):=\alpha s+\frac{1}{2} \omega_{s}
$$

Initially, we would like to calculate some derivatives which will be useful in our analysis later.

$$
\begin{aligned}
& \frac{\partial \phi}{\partial s}=\alpha+\frac{1}{2} \omega_{s}=\alpha+\frac{\cos (\theta) \cos (s)}{\sqrt{1-\sin ^{2}(s) \cos ^{2}(\theta)}} \\
& \frac{\partial^{2} \phi}{\partial s^{2}}=\frac{1}{2} \omega_{s}^{\prime \prime}=\frac{-\sin (s) \cos (\theta) \sin ^{2}(\theta)}{\left(1-\sin ^{2}(s) \cos ^{2}(\theta)\right)^{3 / 2}} \\
& \frac{\partial^{3} \phi}{\partial s^{3}}=\frac{1}{2} \omega_{s}^{\prime \prime \prime}=\frac{-\sin ^{2}(\theta) \cos (\theta) \cos (s)\left(1+2 \sin ^{2}(s) \cos ^{2}(\theta)\right)}{\left(1-\sin ^{2}(s) \cos ^{2}(\theta)\right)^{5 / 2}}
\end{aligned}
$$

\footnotetext{
${ }^{2}$ Note that $t$ is defined to be the total number of steps taken during the walk.
} 
After calculating these derivatives, we need to find an appropriate region in terms of $\alpha$ for which to analyze the asymptotic behavior of the wave function $\Psi$ as $t \rightarrow+\infty$. We would like to choose $\alpha$, so that $I_{1}(\alpha, t)$ and $I_{2}(\alpha, t)$ decay faster than any inverse polynomial in $t$ in the region $|\alpha|+\epsilon$, go as $t^{-1 / 3}$ in the regions around $|\alpha|$, and as $t^{-1 / 2}$ in the third interval [5].

In order to look for stationary points of order 2 around $|\alpha|$, we would like to have $\frac{\partial \phi}{\partial s}=\frac{\partial^{2} \phi}{\partial s^{2}}=0$, but $\frac{\partial^{3} \phi}{\partial s^{3}} \neq 0$.

First off, if

$$
\frac{\partial^{2} \phi}{\partial s^{2}}=\frac{-\sin (s) \cos (\theta) \sin ^{2}(\theta)}{\left(1-\sin ^{2}(s) \cos ^{2}(\theta)\right)^{3 / 2}}=0,
$$

we must have $\sin (s)=0$, so $s=0, \pi$ are the stationary points.

Additionally, solving $\frac{\partial \phi}{\partial s}=0$ for $\alpha$ results in

$$
\alpha=\frac{\mp \cos (\theta) \sqrt{1-\sin ^{2}(s)}}{\sqrt{1-\sin ^{2}(s) \cos ^{2}(\theta)}} .
$$

If $s=0, \pi$, then $\alpha= \pm \cos (\theta)$. However, we must be careful and observe that for $\phi, s=0 \Rightarrow \alpha=-\cos (\theta)$ and $s=\pi \Rightarrow \alpha=\cos (\theta)$.

Now we would like to analyze the behaviors of $I_{1}$ and $I_{2}$ for $|\alpha|=\cos (\theta)$ and $|\alpha|<\cos (\theta)-\epsilon$ for any constant $\epsilon>0$.

We begin with the points $\alpha=\cos (\theta),-\cos (\theta)$ where $\phi$ has stationary points of order 2 at $s=0, \pi$. Therefore we apply the method of stationary phase with $p=3$ to find the leading terms for $I_{1}$ and $I_{2}$.

First, we evaluate $\phi^{(3)}(s, \alpha)$ at $s=0, \pi$ which will be used in the formula for the integral.

$$
\begin{aligned}
& \phi^{(3)}(0, \alpha)=-\sin ^{2}(\theta) \cos (\theta) \\
& \phi^{(3)}(\pi, \alpha)=\sin ^{2}(\theta) \cos (\theta) .
\end{aligned}
$$

Thus, using the above formulas we get 


$$
\begin{aligned}
I_{1,2}(\alpha, t) & \sim \frac{1+(-1)^{\alpha t}}{2} \frac{g_{1,2}(s)}{2 \pi} e^{i(\phi(s, \alpha) t \pm \pi / 6)} \frac{\Gamma\left(\frac{1}{3}\right)}{3}\left[\frac{3 !}{t\left|\phi^{(3)}(s, \alpha)\right|}\right]^{1 / 3} \\
& \sim \frac{1+(-1)^{\alpha t}}{2} \frac{g_{1}(s)}{6 \pi} e^{i(\phi(s, \alpha) t \pm \pi / 6)} \Gamma\left(\frac{1}{3}\right)\left[\frac{6}{\left|t \sin ^{2}(\theta) \cos (\theta)\right|}\right]^{1 / 3}
\end{aligned}
$$

We also need that for $I_{1}, g_{1}(0)=1-\cos (\theta)$ and $g_{1}(\pi)=1+\cos (\theta)$. Similarly, for $I_{2}, g_{2}(0)=\sin (\theta)$ and $g_{2}(\pi)=-\sin (\theta)$. Furthermore, we can find that $\phi(0,-\cos (\theta))=0$ and $\phi(\pi, \cos (\theta))=\pi \cos (\theta)$.

Using all of the above information we can finally write more specific formulas for the leading terms in the integrals $I_{1}$ and $I_{2}$.

$$
\begin{aligned}
I_{1,2}(-\cos (\theta), t) & \sim \frac{1+(-1)^{\alpha t}}{2} \frac{g_{1,2}(0)}{6 \pi} e^{i(\phi(0,-\cos (\theta)) t-\pi / 6)} \Gamma\left(\frac{1}{3}\right)\left[\frac{6}{\left|t \sin ^{2}(\theta) \cos (\theta)\right|}\right]^{1 / 3} \\
& \sim \frac{1+(-1)^{\alpha t}}{2} \frac{g_{1,2}(0)}{6 \pi}\left(\frac{\sqrt{3}}{2}-\frac{1}{2} i\right) \Gamma\left(\frac{1}{3}\right)\left[\frac{6}{\left|t \sin ^{2}(\theta) \cos (\theta)\right|}\right]^{1 / 3}
\end{aligned}
$$

$$
\begin{aligned}
I_{1,2}(\cos (\theta), t) \sim & \frac{1+(-1)^{\alpha t}}{2} \frac{g_{1,2}(\pi)}{6 \pi} e^{i(\phi(\pi, \cos (\theta)) t+\pi / 6)} \Gamma\left(\frac{1}{3}\right)\left[\frac{6}{\left|t \sin ^{2}(\theta) \cos (\theta)\right|}\right]^{1 / 3} \\
\sim & \frac{1+(-1)^{\alpha t}}{2} \frac{g_{1,2}(\pi)}{6 \pi}\left(\cos \left(\pi \cos (\theta) t+\frac{\pi}{6}\right)+i \sin \left(\pi \cos (\theta) t+\frac{\pi}{6}\right)\right) \\
& \times \Gamma\left(\frac{1}{3}\right)\left[\frac{6}{\left|t \sin ^{2}(\theta) \cos (\theta)\right|}\right]^{1 / 3}
\end{aligned}
$$

Now we turn to the interval of most importance to us, $[-\cos (\theta)+\epsilon, \cos (\theta)-\epsilon]$. When $\alpha$ lies in this region we would like to have $\frac{\partial \phi}{\partial s}=0$, but $\frac{\partial^{2} \phi}{\partial s^{2}} \neq 0$, so each of $\phi$ has two stationary points in this region. 
Solving for (B.14) for s gives

$$
s_{\alpha}= \pm \arccos \left(-\frac{\alpha \tan (\theta)}{\sqrt{1-\alpha^{2}}}\right) .
$$

Note that we get two stationary points for each value of alpha, namely $s_{\alpha}$ and $-s_{\alpha}$.

Once again, we can employ the method of stationary phase but this time for $p=2$. In the most general form, we have 3

$$
\begin{aligned}
I_{1,2}(\alpha, t) & \sim \not 2 \times \frac{1+(-1)^{\alpha t}}{2} \frac{g_{1,2}\left(s_{\alpha}\right)}{2 \pi} e^{\left.i\left(\phi\left(s_{\alpha}\right), \alpha\right) t \pm \pi / 4\right)}\left[\frac{2 !}{t\left|\phi^{(2)}\left(s_{\alpha}, \alpha\right)\right|}\right]^{1 / 2} \frac{\Gamma\left(\frac{1}{2}\right)}{\not{2}} \\
& \sim \frac{1+(-1)^{\alpha t}}{2} \frac{\sqrt{2 \pi}}{2 \pi} \cdot \frac{g_{1,2}\left(s_{\alpha}\right)}{\sqrt{t\left|\omega_{s_{\alpha}}^{\prime \prime}\right|}} e^{i\left(\phi\left(s_{\alpha}, \alpha\right) t \pm \pi / 4\right)} \\
& \sim \frac{1+(-1)^{\alpha t}}{2} \frac{g_{1,2}\left(s_{\alpha}\right)}{\sqrt{2 \pi t\left|\omega_{s_{\alpha}}^{\prime \prime}\right|}}\left(\cos \left(\phi\left(s_{\alpha}, \alpha\right) t+\frac{\pi}{4}\right) \pm \sin \left(\phi\left(s_{\alpha}, \alpha\right) t+\frac{\pi}{4}\right)\right)
\end{aligned}
$$

which can be simplified to

$$
I_{1,2}(\alpha, t) \sim \frac{g_{1,2}\left(s_{\alpha}\right)}{\sqrt{2 \pi t\left|\omega_{s_{\alpha_{1,2}}}^{\prime \prime}\right|}} \times\left\{\begin{array}{lc}
2 \cos \left(\phi\left(s_{\alpha}, \alpha\right) t+\pi / 4\right) & \text { if } g \text { is even } \\
2 i \sin \left(\phi\left(s_{\alpha} \alpha\right) t+\pi / 4\right) & \text { if } g \text { is odd }
\end{array}\right.
$$

The phase is

$$
\phi\left( \pm s_{\alpha}, \alpha\right)= \pm \alpha s_{\alpha}+\frac{1}{2} \omega_{s_{\alpha}}
$$

where

$$
\omega\left( \pm s_{\alpha}\right)= \pm 2 \arcsin \left(\sqrt{\frac{\cos ^{2}(\theta)-\alpha^{2}}{1-\alpha^{2}}}\right)
$$

and the second derivative is

\footnotetext{
${ }^{3}$ Note that $\Gamma\left(\frac{1}{2}\right)=\sqrt{\pi}$.
} 


$$
\begin{aligned}
\frac{\partial^{2}}{\partial s^{2}}\left( \pm s_{\alpha}, \alpha\right) & =\frac{-\sin \left( \pm s_{\alpha}\right) \sin ^{2}(\theta) \cos (\theta)}{\left(1-\sin ^{2}\left( \pm s_{\alpha}\right) \cos ^{2}(\theta)\right)^{3 / 2}} \\
& =\mp \sin ^{2}(\theta) \cos (\theta) \sqrt{\frac{1-\alpha^{2} \sec ^{2}(\theta)}{1-\alpha^{2}}}\left(\frac{\sin ^{2}(\theta)}{1-\alpha^{2}}\right)^{-3 / 2} \\
& =\frac{\mp\left(1-\alpha^{2}\right) \sqrt{\cos ^{2}(\theta)-\alpha^{2}}}{\sin (\theta)} .
\end{aligned}
$$

Therefore, for $\left|\omega_{s_{\alpha}}^{\prime \prime}\right|$ we can just use

$$
\left|\omega_{s_{\alpha}}^{\prime \prime}\right|=\frac{\left(1-\alpha^{2}\right) \sqrt{\cos ^{2}(\theta)-\alpha^{2}}}{|\sin (\theta)|} .
$$

Next, we find $g_{1}\left( \pm s_{\alpha}\right)$ and $g_{2}\left( \pm s_{\alpha}\right)$.

$$
\begin{aligned}
& g_{1}\left( \pm s_{\alpha}\right)=(1+\alpha) \\
& g_{2}\left( \pm s_{\alpha}\right)=-\alpha \tan (\theta) \pm i \sqrt{1-\alpha^{2} \sec ^{2}(\theta)}
\end{aligned}
$$

Combining the aforementioned calculations, we can write an asymptotic expression given in 13 for $a_{j}(\alpha t, t)$ and $b_{j}(\alpha t, t)$, where $a_{j}(\alpha t, t):=I_{1}$ and $b_{j}(\alpha t, t):=I_{2}$.

\section{Numerical Results}

\section{C.1. Rotations Walk Numerical Simulations}

Below we present numerical simulations for 100 time steps for the alternating Rotations quantum walk with initial condition $|\uparrow\rangle \otimes|0\rangle$ for various values of $\theta$, $\theta \in[0, \pi]$. Note that only the points for even positions are graphed as the probabilities at odd positions are zero. The vertical dashed lines mark the stationary points of order 2 which occur at $\pm \cos (\theta) t$. 

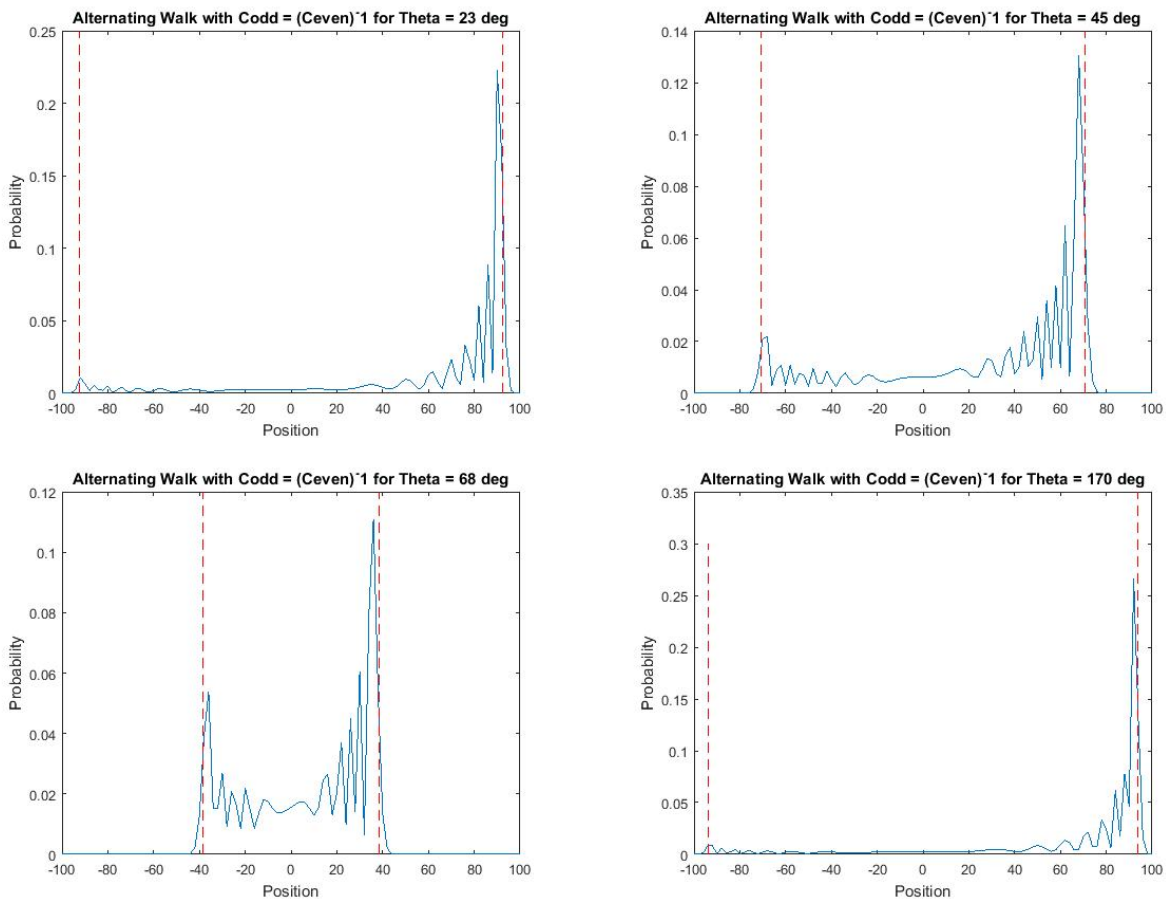